\title{
SIGMA, TAU AND ABELIAN FUNCTIONS OF ALGEBRAIC CURVES
}

\author{
J. C. EILBECK, V.Z. ENOLSKI, AND J.GIBBONS
}

\begin{abstract}
We compare and contrast three different methods for the construction of the differential relations satisfied by the fundamental Abelian functions associated with an algebraic curve. We realize these Abelian functions as logarithmic derivatives of the associated sigma function. In two of the methods, the use of the tau function, expressed in terms of the sigma function, is central to the construction of differential relations between the Abelian functions.
\end{abstract}

\section{INTRODUCTION}

In the mid-1970s, the results of Its, Matveev, Dubrovin and Novikov (see [9]) led to the discovery of a remarkable $\theta$-functional formula to solve the KdV equation $u_{t}=6 u u_{x}-u_{x x x}$. This solution was given as a second logarithmic derivative of a Riemann theta-function:

$$
u(x, t)=-\frac{\partial^{2}}{\partial x^{2}} \ln \theta(\boldsymbol{U} x+\boldsymbol{V} t+\boldsymbol{W})+C
$$

with $\boldsymbol{U}, \boldsymbol{V}, \boldsymbol{W}=$ const $\in \mathbb{C}^{g}$ and $C \in \mathbb{C}$. The theta-function in that formula was constructed from a hyperelliptic curve $X_{g}$ of genus $g$, while the "winding vectors" $\boldsymbol{U}, \boldsymbol{V}$ are periods of abelian differentials of the second kind on $X_{g}$. Further, this formula is in a sense universal; it was generalized by Krichever [20] to other integrable hierarchies - whose solutions were associated with other algebraic curves. In this paper we consider the converse problem:

Given an algebraic curve $X_{g}$, of genus $g$, its Riemann period matrix $\tau$, and its Jacobi variety $\operatorname{Jac}\left(X_{g}\right)=\mathbb{C} /\left(1_{g} \oplus \tau\right)$, we may construct $\theta$-functions $\theta(\boldsymbol{u} ; \tau)$, $\boldsymbol{u} \in \operatorname{Jac}\left(X_{g}\right)$; then the fundamental Abelian functions on $\operatorname{Jac}\left(X_{g}\right)$ may be realized as the second logarithmic derivatives of $\theta(\boldsymbol{u}, \tau), \wp_{i j}(\boldsymbol{u})=-\frac{\partial^{2} \ln \theta(\boldsymbol{u} ; \tau)}{\partial u_{i} \partial u_{j}}$. We wish to construct all differential relations between these Abelian functions on $\operatorname{Jac}\left(X_{q}\right)$.

The simplest case, the Weierstrass cubic, $y^{2}=4 x^{3}-g_{2} x-g_{3}$, is an algebraic curve of genus one. This is uniformized by the Weierstrass elliptic functions,

Date: September 11, 2018. 
$x=\wp(u), y=\wp^{\prime}(u)$, and the differential relations are

$$
\wp^{\prime \prime}=6 \wp^{2}-\frac{g_{2}}{2}, \quad \wp^{\prime 2}=4 \wp^{3}-g_{2} \wp-g_{3} .
$$

In the case of higher genera, $g>1$, the derivation of analogous equations becomes much more complicated. In particular, the fundamental Abelian functions are now partial derivatives of a function of $g$ variables. The different approaches to this problem form the main content of the paper. We restrict our analysis to the case of $(n, s)$-curves introduced and investigated in this context by Buchstaber, Leykin and Enolski [3, 4]

$$
X_{g}: y^{n}=x^{s}+\sum_{n i+s j<n s} \lambda_{i j} x^{i} y^{j} .
$$

These represent a natural generalization of elliptic curves to higher genera, and include the general hyperelliptic curve $(n=2)$.

To any such curve we may associate an object which is fundamental to all our treatments of this problem, the fundamental bi-differential. This is the unique symmetric meromorphic 2 -form on $X_{g} \times X_{g}$, whose only second order pole lies on the diagonal $Q=S$, and which satisfies

$$
\omega(Q, S)-\frac{\mathrm{d} \xi(Q) \mathrm{d} \xi(S)}{(\xi(Q)-\xi(S))^{2}}=\Phi(\xi(S), \xi(Q)) \mathrm{d} \xi(Q) \mathrm{d} \xi(S)
$$

where $\Phi(\xi(S), \xi(Q))$ is holomorphic, and $\xi(Q), \xi(S)$ are local coordinates in the vicinity of a base point $P, \xi(P)=0$. Usually $\omega(Q, S)$ is realized as the second logarithmic derivative of the prime-form or theta-function [14. But in our development we use an alternative representation of $\omega(Q, S)$ in the algebraic form that goes back to Weierstrass and Klein, and which was well documented by Baker [1]

$$
\omega(Q, S)=\frac{\mathcal{F}(Q, S)}{f_{y}(Q) f_{w}(S)(x-z)^{2}} \mathrm{~d} x \mathrm{~d} z+2 \mathrm{~d} \boldsymbol{u}(Q)^{T} \varkappa \mathrm{d} \boldsymbol{u}(S),
$$

where $Q=(x, y), S=(z, w)$, and the function $\mathcal{F}(Q, S)=\mathcal{F}((x, y),(z, w))$ is a polynomial of its arguments with coefficients depending on the parameters of the curve $X_{g} \mathrm{~d} \boldsymbol{u}=\left(\mathrm{d} u_{1}, \ldots, \mathrm{d} u_{g}\right)^{T}$ is the vector of basic holomorphic differentials. The factor 2 in the final term is introduced so that this theory reduces to the Weierstrass theory in the elliptic case. Here, $\varkappa$ is a symmetric matrix expressed in terms of the first and second period matrices, $2 \omega, 2 \eta$ respectively, as $\varkappa=\omega^{-1} \eta$. This provides the normalization of $\omega(Q, S)$. We will refer to the first term on the right of (1.4), which involves the polynomial $\mathcal{F}(Q, S)$, as the algebraic part, so that

$$
\omega(Q, S)=\omega^{\mathrm{alg}}(Q, S)+2 \mathrm{~d} \boldsymbol{u}(Q)^{T} \varkappa \mathrm{d} \boldsymbol{u}(S) .
$$


This representation was revisited and developed by Buchstaber, Leykin and Enolski [3] and more recently by Nakayashiki [23].

The algebraic representation of the fundamental differential, as described above, lies behind the definition of the multivariate sigma function in terms of the theta-function. This differs from $\theta$ by an exponential factor and a modular factor:

$$
\sigma(\boldsymbol{u})=C(\tau) \exp \left\{\frac{1}{2} \boldsymbol{u}^{T} \omega^{-1} \eta \boldsymbol{u}\right\} \theta\left(\frac{1}{2} \omega^{-1} \boldsymbol{u} ; \tau\right) .
$$

Here the $g \times g$ matrices $2 \omega, 2 \eta$ are the first and second period matrices, and $\tau=\omega^{-1} \omega^{\prime}$. The modular constant $C(\tau)$ is known explicitly for hyperelliptic curves and a number of other cases. However its explicit form is not necessary here, for the fundamental Abelian functions are independent of $C(\tau)$. These modifications make $\sigma(\boldsymbol{u})$ invariant with respect to the action of the symplectic group, so that for any $\gamma \in \operatorname{Sp}(2 g, \mathbb{Z})$, we have:

$$
\sigma(\boldsymbol{u} ; \gamma \tau)=\sigma(\boldsymbol{u} ; \tau)
$$

The multivariate sigma-function is the natural generalization of the Weierstrass sigma function to algebraic curves of higher genera, i.e. $(n, s)$-curves in this context. In his lectures [30], Weierstrass started by defining the sigmafunction in terms of series with coefficients given recursively, which was the key point of the Weierstrass theory of elliptic functions. A generalization of this result to the genus two curve was started by Baker [2] and recently completed by Buchstaber and Leykin [6], who obtained recurrence relations between coefficients of the sigma-series in closed form. In addition, Buchstaber and Leykin recently found an operator algebra that annihilates the sigma-function of a higher genera $(n, s)$-curve [7]. The recursive definition of the higher genera sigma-functions remains a challenging problem to solve, with [7] providing a definite step. We believe that the future theory of the sigma and corresponding Abelian functions can be formulated on the basis of sigma expansions that will complete the extension of the Weierstrass theory to curves of higher genera.

In this paper we study the interrelation of the multivariate sigma and Sato tau functions. The $\tau$-function was introduced by Sato [28, 29] in the much more general context of integrable hierarchies. But it seems there are few results studying algebraic curves in Sato theory. However we should mention recent work by Konopelchenko and Ortenzi analyzing algebro-geometric structure in Birkhoff strata of the Sato Grassmannian [19]. The recent papers of Matsutani and Previato [21, 22], studying Jacobi inversion on Jacobian strata of $(r, s)$ curves, is also relevant to our work, relating stratification of the Sato Grassmannian to partitions.

Here we deal with the 'algebro-geometric $\tau$-function' (AGT) associated with an algebraic curve. 
The AGT of the genus $g$ curve $X_{g}$ is defined, following Fay, [15, 16], as a function of the 'times' $\boldsymbol{t}=\left(t_{1}, \ldots, t_{g}, t_{g+1}, \ldots\right)$, a point $\boldsymbol{u} \in \operatorname{Jac}\left(X_{g}\right)$, as well as a point $P \in X_{g}$; it is given by the formula

$$
\tau(\boldsymbol{t} ; \boldsymbol{u}, P)=\theta\left(\sum_{k=1}^{\infty} \boldsymbol{U}_{k}(P) t_{k}+\frac{1}{2} \omega^{-1} \boldsymbol{u}\right) \exp \left\{\frac{1}{2} \sum_{m, n \geq 1} \omega_{m n}(P) t_{m} t_{n}\right\} .
$$

Here the "winding vectors" $\boldsymbol{U}_{k}(P)$ appear in the expansion of the normalized holomorphic integral $\boldsymbol{v}$, the quantities $\omega_{m n}(P)$ define the holomorphic part of the expansion of the fundamental differential of the second kind $\omega(Q, S)$ near the point $P$. We then introduce the $\tau$-function by the formula:

$$
\frac{\tau(\boldsymbol{t} ; \boldsymbol{u}, P)}{\tau(\mathbf{0} ; \boldsymbol{u}, P)}=\frac{\sigma\left(\sum_{k=1}^{\infty} \mathcal{A}^{-1} \boldsymbol{U}_{k}(P) t_{k}+\boldsymbol{u}\right)}{\sigma(\boldsymbol{u})} \exp \left\{\frac{1}{2} \sum_{k, l=0}^{\infty} \omega_{k, l}^{\mathrm{alg}}(P) t_{k} t_{l}\right\} \text {. }
$$

This representation of $\tau$ in terms of $\sigma$ was used by Harnad and Enolski [13. to analyze the Schur function expansion of $\tau$ for the case of algebraic curves. Recently Nakayashiki [25] has independently suggested a similar expression for the AGT in terms of multivariate $\sigma$-functions. In this paper we concentrate on the application of this representation to the derivation of the differential relations between Abelian functions of the $(n, s)$-curve, continuing and developing the work of [13].

Developing a further analogy with the Weierstrass theory of elliptic functions, we represent the Abelian functions, that is, $2 g$-periodic functions

$$
F\left(\boldsymbol{u}+2 \boldsymbol{n} \omega+2 \boldsymbol{n}^{\prime} \omega^{\prime}\right)=F(\boldsymbol{u}), \quad \forall \boldsymbol{n}, \boldsymbol{n}^{\prime} \in \mathbb{N}
$$

on the Jacobian

$$
\widetilde{\operatorname{Jac}}\left(X_{g}\right)=\mathbb{C} / 2 \omega \oplus 2 \omega^{\prime}=\mathcal{A}^{-1} \operatorname{Jac}\left(X_{g}\right), \quad 2 \omega=\mathcal{A},
$$

as second and higher logarithmic derivatives:

$$
\begin{aligned}
\zeta_{i}(\boldsymbol{u}) & =\frac{\partial}{\partial u_{i}} \ln \sigma(\boldsymbol{u}), \\
\wp_{i j}(\boldsymbol{u}) & =-\frac{\partial^{2}}{\partial u_{i} \partial u_{j}} \ln \sigma(\boldsymbol{u}), \quad \wp_{i j k}(\boldsymbol{u})=-\frac{\partial^{3}}{\partial u_{i} \partial u_{j} \partial u_{k}} \ln \sigma(\boldsymbol{u}), \quad \text { etc. },
\end{aligned}
$$

where $i, j, k, \cdots=1 \ldots, g$. We should remark that the $\zeta_{i}(\boldsymbol{u})$ are not Abelian functions. In this notation, the genus one Weierstrass equations (1.2) become

$$
\wp_{1111}=6 \wp_{11}^{2}-\frac{g_{2}}{2}, \quad \wp_{111}^{2}=4 \wp_{11}^{3}-g_{2} \wp_{11}-g_{3} .
$$

For general $g$, the $\wp_{i j}, \wp_{i j k}, \ldots$ are called Kleinian $\wp$-functions. They are convenient coordinates to represent the dependent variables in the hierarchy of integrable systems. 
In this paper we compare and contrast three approaches to obtain the partial differential relations for the Abelian functions associated with the $(n, s)$-curve $X_{g}$, using Kleinian $\wp$-functions as coordinates.

The first of these, and the best known, is the classical approach of comparing two different expansions of the fundamental bi-differential; this yields first the solution of the Jacobi inversion problem for the curve, and in higher orders, a sequence of differential relations involving the $\wp_{i j}$.

The $\tau$-function approach to the derivation of completely integrable systems of KP type has led to two different ways [8] to obtain relations between Taylor coefficients of the $\tau$ function expansion. The first of these specifically exploits the fact that these Taylor coefficients are determinants, i.e. Plücker coordinates in the Grassmanian, and they hence satisfy the Plücker relations [15. The second is based on the Bilinear Identity, which leads to the Residue Formula [16], giving a family of differential polynomials in $\tau$ which must vanish - these are the partial differential equations we need.

We further consider and compare the two techniques based on the $\tau$-function method, which give a derivation of the required differential relations; specializing to a particular algebraic curve, we consider its algebro-geometric $\tau$ function. The special feature of our development is that we define this $\tau$ function in terms of the multidimensional $\sigma$-function of the curve, leading to coordinates that are explicitly written in terms of Kleinian $\wp$-functions. The differential relations we find between these functions can be understood as arising from special solutions of integrable hierarchies of KP type, associated with the given curve (see for example [1, 3, 23]). We will describe the correspondence between individual differential equations for $\wp$-functions with Young diagrams defining Plücker relations. We illustrate these approaches by considering two particular examples: the genus 2 hyperelliptic curves [2], and the genus 3 trigonal curve, which can be found in [3, 5, 12]).

We remark that in similar context, Nimmo [26] in 1982 applied the methods of symmetric function theory to describe the KP hierarchy, and studied the action of the recursion operator. The general approach based on Plücker coordinates for deriving KP-flows in terms of Kleinian $\sigma$-functions was recently discussed in [13. Here we develop these ideas and consider some non-trivial examples to clarify the interrelation of the $\tau$-functional formulation of integrable hierarchies and the $\sigma$-functional approach. We will also consider the relationship between of this derivation, based on the Plücker relations, and that based on the Residue Formula. Both give a systematic way of generating the required relations, but the differences between the two approaches are instructive. 


\section{Algebraic Curves}

Let $X_{g}$ be a genus $g \geq 1$ algebraic curve given by the polynomial equation

$$
f(x, y)=0, \quad f(x, y)=y^{n}+y^{n-1} a_{1}(x)+\ldots+a_{0}(x) .
$$

We shall consider in what follows two relatively simple curves of the class (2.1),

Example I: the hyperelliptic genus two curve

$$
y^{2}=4 x^{5}+\alpha_{4} x^{4}+\ldots+\alpha_{0}
$$

and

Example II: the cyclic trigonal genus three curve

$$
f(x, y)=y^{3}-\left(x^{4}+\mu_{3} x^{3}+\mu_{6} x^{2}+\mu_{9} x+\mu_{12}\right) .
$$

We equip $X_{g}$ with a canonical basis of cycles $\left(\mathfrak{a}_{1}, \ldots, \mathfrak{a}_{g} ; \mathfrak{b}_{1}, \ldots, \mathfrak{b}_{g}\right) \in H_{1}(X, \mathbb{Z})$. We denote by $\mathrm{d} \boldsymbol{u}=\left(\mathrm{d} u_{1}, \ldots, \mathrm{d} u_{g}\right)^{T}$ the vector whose entries are independent holomorphic differentials of the curve $X_{g}$ as well as their $\mathfrak{a}$ and $\mathfrak{b}$-periods,

$$
2 \omega=\left(\oint_{\mathfrak{a}_{j}} \mathrm{~d} u_{i}\right)_{i, j=1, \ldots, g}, 2 \omega^{\prime}=\left(\oint_{\mathfrak{b}_{j}} \mathrm{~d} u_{i}\right)_{i, j=1, \ldots, g}
$$

The period matrix $\left(2 \omega, 2 \omega^{\prime}\right)$ is the first period matrix, and the matrix $\tau=$ $\omega^{-1} \omega^{\prime}$ belongs to the upper Siegel half-space, $\mathfrak{S}: \tau^{T}=\tau, \operatorname{Im} \tau>0$.

The $\theta$-function $\theta[\alpha](\boldsymbol{z} ; \tau)$ with characteristics $[\alpha]=\left[\begin{array}{l}\boldsymbol{a}^{T} \\ \boldsymbol{b}^{T}\end{array}\right],[2 \alpha] \in \mathbb{Z}^{g} \times \mathbb{Z}^{g}$ of the algebraic curve $X_{g}$ of genus $g$ is defined through its Fourier series

$$
\theta\left[\begin{array}{l}
\boldsymbol{a}^{T} \\
\boldsymbol{b}^{T}
\end{array}\right](\boldsymbol{z} ; \tau)=\sum_{\boldsymbol{m} \in \mathbb{Z}^{g}} \exp \left\{\imath \pi(\boldsymbol{m}+\boldsymbol{a})^{T} \tau(\boldsymbol{m}+\boldsymbol{a})+2 \imath \pi(\boldsymbol{m}+\boldsymbol{a})^{T}(\boldsymbol{z}+\boldsymbol{b})\right\}
$$

and possesses the periodicity property: for arbitrary $\boldsymbol{a}, \boldsymbol{b} \in \mathbb{Q}^{g}$ and arbitrary $\boldsymbol{a}^{\prime}, \boldsymbol{b}^{\prime} \in \mathbb{Q}^{g}$ the following formula is valid

$$
\begin{aligned}
& \theta\left[\begin{array}{c}
\boldsymbol{a}^{T} \\
\boldsymbol{b}^{T}
\end{array}\right]\left(\boldsymbol{z}+\tau \boldsymbol{a}^{\prime}+\boldsymbol{b}^{\prime} ; \tau\right) \\
& =\theta\left[\left(\boldsymbol{a}+\boldsymbol{a}^{\prime}\right)^{T}\left(\boldsymbol{b}+\boldsymbol{b}^{\prime}\right)^{T}\right](\boldsymbol{z} ; \tau) \exp \left\{-\imath \pi \boldsymbol{a}^{\prime T} \tau \boldsymbol{a}^{\prime}-2 \imath \pi \boldsymbol{a}^{\prime T} \boldsymbol{z}-2 \imath \pi\left(\boldsymbol{b}+\boldsymbol{b}^{\prime}\right)^{T} \boldsymbol{a}^{\prime}\right\}
\end{aligned}
$$

We introduce the associated meromorphic differentials $\mathrm{d} \boldsymbol{r}=\left(\mathrm{d} r_{1}, \ldots, \mathrm{d} r_{g}\right)^{T}$ and their periods

$$
2 \eta=-\left(\oint_{\mathfrak{a}_{j}} \mathrm{~d} r_{i}\right)_{i, j=1, \ldots, g}, \quad 2 \eta^{\prime}=-\left(\oint_{\mathfrak{b}_{j}} \mathrm{~d} r_{i}\right)_{i, j=1, \ldots, g}
$$


which form the second period matrix $\left(2 \eta, 2 \eta^{\prime}\right)$. The period matrices satisfy the condition

$$
\left(\begin{array}{cc}
\omega & \omega^{\prime} \\
\eta & \eta^{\prime}
\end{array}\right)\left(\begin{array}{cc}
0 & 1_{g} \\
-1_{g} & 0
\end{array}\right)\left(\begin{array}{cc}
\omega & \omega^{\prime} \\
\eta & \eta^{\prime}
\end{array}\right)^{T}=-\frac{\imath \pi}{2}\left(\begin{array}{cc}
0 & 1_{g} \\
-1_{g} & 0
\end{array}\right)
$$

Here we denote the half-periods of the holomorphic and meromorphic differentials by $\left(\omega, \omega^{\prime}\right)$ and $\left(\eta, \eta^{\prime}\right)$ in order to emphasize the analogy with the Weierstrass theory. We will also use the notation $\mathcal{A}=2 \omega$ and $\mathcal{B}=2 \omega^{\prime}$ for the periods of holomorphic differentials. Further we denote

$$
\mathrm{d} \boldsymbol{v}(Q)=\left(\mathrm{d} v_{1}(Q), \ldots, \mathrm{d} v_{g}(Q)\right)^{T}=\mathcal{A}^{-1} \mathrm{~d} \boldsymbol{u}(Q)
$$

as the vector of normalized holomorphic differentials.

The explicit calculation of canonical holomorphic differentials and the meromorphic differentials conjugate to them is well understood; in particular we have

Example I

$$
\begin{aligned}
& \mathrm{d} u_{1}=\frac{x \mathrm{~d} x}{y}, \quad \mathrm{~d} u_{2}=\frac{\mathrm{d} x}{y}, \\
& \mathrm{~d} r_{1}=\frac{x^{2} \mathrm{~d} x}{y}, \quad \mathrm{~d} r_{2}=\frac{x\left(\alpha_{3}+2 \alpha_{4} x+12 x^{2}\right) \mathrm{d} x}{4 y} ;
\end{aligned}
$$

Example II

$$
\begin{aligned}
& \mathrm{d} u_{1}=\frac{\mathrm{d} x}{3 y}, \quad \mathrm{~d} u_{2}=\frac{x \mathrm{~d} x}{3 y^{2}}, \quad \mathrm{~d} u_{3}=\frac{\mathrm{d} x}{3 y^{2}}, \\
& \mathrm{~d} r_{1}=\frac{x^{2} \mathrm{~d} x}{3 y^{2}}, \quad \mathrm{~d} r_{2}=-\frac{2 x \mathrm{~d} x}{3 y}, \quad \mathrm{~d} r_{3}=-\frac{\left(5 x^{2}+3 \mu_{3} x+\mu_{6}\right)}{3 y} \mathrm{~d} x .
\end{aligned}
$$

Remark 2.1. Note that our labelling of the differentials is the reverse of [12], with the interchange $1 \leftrightarrow 2$ in example I, and $(1,2,3) \leftrightarrow(3,2,1)$ in example II.

We introduce the fundamental bi-differential $\omega(Q, S)$ on $X \times X$ which is uniquely defined by the conditions:

(i) it is symmetric:

$$
\omega(Q, S)=\omega(S, Q)
$$

(ii) it has its only pole along the diagonal $Q=S$, in which neighbourhood it is expanded in a power series according to

$$
\omega(Q, S)-\frac{\mathrm{d} \xi(Q) \mathrm{d} \xi(S)}{(\xi(Q)-\xi(S))^{2}}=\sum_{m, n \geq 1} \omega_{m n}(P) \xi(Q)^{m-1} \xi(S)^{n-1} \mathrm{~d} \xi(Q) \mathrm{d} \xi(S)
$$


(iii) it is normalized such that:

$$
\oint_{\mathfrak{a}_{j}} \omega(Q, S)=0, \quad j=1, \ldots, g .
$$

The well known realization of the differential $\omega(Q, S)$ involves the SchottkyKlein prime form $E(Q, S)$, which is a $(-1 / 2,-1 / 2)$-differential defined for arbitrary points $Q, S \in X$

$$
E(Q, S)=\frac{\theta[\alpha]\left(\int_{Q}^{S} \mathrm{~d} \boldsymbol{v}\right)}{h_{\alpha}(Q) h_{\alpha}(S)}
$$

where $\theta[\alpha](\boldsymbol{u})$ is a $\theta$-function with non-singular odd characteristics $[\alpha], \mathrm{d} \boldsymbol{v}$ is the vector of normalized holomorphic differentials, and

$$
h_{\alpha}(Q)^{2}=\sum_{k=1}^{g} \frac{\partial}{\partial z_{k}} \theta[\alpha](\mathbf{0}) \mathrm{d} v_{k}(Q)
$$

The bi-differential $\omega(Q, S)$ is then given by [14].

$$
\omega(Q, S)=\mathrm{d}_{Q} \mathrm{~d}_{S} \ln E(Q, S) .
$$

We emphasize that in this paper, we will instead rely on alternative "algebraic" constructions of the differential $\omega(Q, S)$. By following classical works such as [17, 18], together with results documented in [1] we express the differential $\omega(Q, S)$ in the form

$$
\omega(Q, S)=\frac{\mathcal{F}(Q, S)}{f_{y}(Q) f_{w}(S)(x-z)^{2}} \mathrm{~d} x \mathrm{~d} z+2 \mathrm{~d} \boldsymbol{u}(Q)^{T} \varkappa \mathrm{d} \boldsymbol{u}(S),
$$

where $Q=(x, y), S=(z, w)$, and the function $\mathcal{F}(Q, S)=\mathcal{F}((x, y),(z, w))$ is a polynomial of its arguments, with coefficients depending on the moduli of the curve $X_{g}$. Finally, $\varkappa$ is a symmetric matrix $\varkappa^{T}=\varkappa$ that is chosen to provide a normalization of $\omega(Q, S)$; it is expressible in terms of the first and second period matrices $\varkappa=\omega^{-1} \eta$. We will refer to the term of $\omega(Q, S)$ including the polynomial $\mathcal{F}(Q, S)$ as its algebraic part,

$$
\omega(Q, S)=\omega^{\operatorname{alg}}(Q, S)+2 \mathrm{~d} \boldsymbol{u}(Q)^{T} \varkappa \mathrm{d} \boldsymbol{u}(S)
$$

In the vicinity of a base point $P$, where points $Q$ and $S$ are represented by local coordinates $\xi(Q)$ and $\xi(S)$ respectively, the holomorphic part of $\omega^{\text {alg }}(Q, S)$ is expanded in the series

$$
\begin{aligned}
& \left.\frac{\mathcal{F}(Q, S) \mathrm{d} z \mathrm{~d} x}{f_{y}(Q) f_{w}(S)(x-z)^{2}}\right|_{x=x(Q), z=x(S)}-\frac{\mathrm{d} \xi(Q) \mathrm{d} \xi(S)}{(\xi(Q)-\xi(S))^{2}} \\
& =\sum_{k, l=0}^{\infty} \omega_{k, l}^{\mathrm{alg}}(P) \xi(Q)^{k} \xi(S)^{l} \mathrm{~d} \xi(Q) \mathrm{d} \xi(S) .
\end{aligned}
$$


An algorithm to construct the polynomial $\mathcal{F}(Q, S)$ is known, see e.g. [1] and therefore functions such as $\omega_{k, l}^{\text {alg }}(P)$ are needed for our construction are considered as known. In all that follows we will take the fixed base point $P$ to be $(\infty, \infty)$. We shall present below some explicit expressions for $\mathcal{F}$ as well as the first few terms of the expansions $\omega_{k, l}^{\text {alg }}(P)$ in the simplest cases:

Example I:

$$
\omega^{\operatorname{alg}}(Q, S)=\frac{F(x, z)+2 y w}{4(x-z)^{2}} \frac{\mathrm{d} x}{y} \frac{\mathrm{d} z}{w}, \quad Q=(x, y), S=(z, w)
$$

where

$$
F(x, z)=4 x^{2} z^{2}(x+z)+2 \alpha_{4} x^{2} z^{2}+\alpha_{3} x z(x+z)+2 \alpha_{2} x z+\alpha_{1}(x+z)+2 \alpha_{0} .
$$

The polynomial $F(x, z)$, with the properties $F(x, z)=F(z, x)$ and $F(x, x)=$ $2 y^{2}$, is sometimes called the "Kleinian polar".

Expanding this about the base point gives:

$$
\begin{aligned}
\omega_{0,0}^{\mathrm{alg}} & =-\frac{\alpha_{4}}{8} \\
\omega_{0,1}^{\mathrm{alg}} & =\omega_{1,0}^{\mathrm{alg}}=0, \\
\omega_{0,2}^{\mathrm{alg}} & =\omega_{2,0}^{\mathrm{alg}}=-\frac{16 \alpha_{3}-3 \alpha_{4}^{2}}{128}, \quad \omega_{1,1}^{\mathrm{alg}}=0, \\
& \ldots \text { etc. }
\end{aligned}
$$

Remark 2.2. For hyperelliptic curves, the coefficients $\omega_{i, j}^{\text {alg }}$ vanish if either of $i$ or $j$ is odd.

\section{Example II:}

$$
\omega^{\mathrm{alg}}((x, y),(z, w))=\frac{\mathcal{F}((x, y),(z, w)) d x d z}{(x-z)^{2} f_{y}(x, y) f_{w}(z, w)}
$$

with the polynomial $\mathcal{F}((x, y) ;(z, w))$ given by the formula

$$
\begin{aligned}
& \mathcal{F}((x, y),(z, w))=w^{2} y^{2} \\
& \quad+w\left(w\left[\frac{f(x, y)}{y}\right]_{y}+T(x, z)\right)+y\left(y\left[\frac{f(z, w)}{w}\right]_{w}+T(z, x)\right)
\end{aligned}
$$

and

$$
\begin{aligned}
T(x, z) & =3 \mu_{12}+(z+2 x) \mu_{9}+x(x+2 z) \mu_{6} \\
& +3 \mu_{3} x^{2} z+x^{2} z^{2}+2 x^{3} z .
\end{aligned}
$$

Expanding this about the base point gives:

$$
\omega_{0,0}^{\mathrm{alg}}=0,
$$




$$
\begin{aligned}
& \omega_{0,1}^{\mathrm{alg}}=\omega_{1,0}^{\mathrm{alg}}=-\frac{2}{3} \mu_{3}, \\
& \omega_{0,4}^{\mathrm{alg}}=\omega_{4,0}^{\mathrm{alg}}=-\frac{2}{3} \mu_{6}+\frac{5}{9} \mu_{3}^{2}, \\
& \omega_{1,3}^{\mathrm{alg}}=\omega_{3,1}^{\mathrm{alg}}=-\frac{2}{3} \mu_{6}+\frac{4}{9} \mu_{3}^{2}, \\
& \omega_{2,2}^{\mathrm{alg}}=0, \\
& \ldots \text { etc. }
\end{aligned}
$$

Remark 2.3. $\omega_{i, j}^{\mathrm{alg}}=0$ unless $i+j \equiv 1 \bmod 3$. This is a consequence of the cyclic symmetry of the curve.

\section{Algebro-GeOmetric $\theta, \sigma$ And $\tau$-FUnCtions}

Let $\boldsymbol{v} \in \operatorname{Jac}\left(X_{g}\right)$ and $\theta(\boldsymbol{v})$ be a canonical $\theta$-function, that is, a $\theta$-function with zero characteristics:

$$
\theta(\boldsymbol{v})=\sum_{\boldsymbol{m} \in \mathbb{Z}^{g}} \exp \left\{\imath \pi \boldsymbol{m}^{T} \tau \boldsymbol{m}+2 \imath \pi \boldsymbol{v}^{T} \boldsymbol{m}\right\} .
$$

The starting point of this paper is the transition from $\theta$ to $\sigma$-functions. For any point $\boldsymbol{u} \in \operatorname{Jac}(X)$ we define:

$$
\sigma(\boldsymbol{u})=C(\tau) \theta\left(\mathcal{A}^{-1} \boldsymbol{u}\right) \exp \left\{\frac{1}{2} \boldsymbol{u}^{T} \varkappa \boldsymbol{u}\right\}
$$

where $\theta(\boldsymbol{v})$ is the canonical $\theta$-function, and $C(\tau)$ is a certain modular constant that we do not need for the results that follow. We note that this $\sigma$-function differs from the "fundamental $\sigma$ function" of the publications mentioned in the introduction by the absence of a shift of the $\theta$-argument by the vector of Riemann constants. Thus $\sigma(\mathbf{0}) \neq 0$. The sigma-function inherits a quasiperiodicity property from the corresponding $\theta$-function.

We introduce the Kleinian multi-variable $\zeta$ and $\wp$-functions as above (1.9, 1.10). These functions are suitable coordinates to describe Abelian functions and the KP-type hierarchies of differential relations between them.

In higher genera, the relations between Abelian functions become somewhat lengthy. These relations can often be summarized concisely by developing a matrix formulation of the theory, as was done [3] in the hyperelliptic case.

The Sato-Fay algebro-geometric $\tau$-function of the genus $g$ curve $X_{g}$ of arguments $\boldsymbol{t}=\left(t_{1}, \ldots, t_{g}, t_{g+1}, \ldots\right)^{T}, \boldsymbol{u} \in \operatorname{Jac}(X), P \in X_{g}$ is defined as

$$
\tau(\boldsymbol{t} ; \boldsymbol{u}, P)=\theta\left(\sum_{k=1}^{\infty} \boldsymbol{U}_{k}(P) t_{k}+\mathcal{A}^{-1} \boldsymbol{u}\right) \exp \left\{\frac{1}{2} \sum_{m, n \geq 1} \omega_{m n}(P) t_{m} t_{n}\right\} .
$$


Here $\mathcal{A}$ is the matrix of periods of canonical holomorphic differentials and the winding vectors defined in (3.3), $\boldsymbol{U}_{k}(P)$, appear in the expansion of the normalized holomorphic integral $\boldsymbol{v}$ in the vicinity of the given point $P \in X$,

$$
\int_{P_{0}}^{Q} \mathrm{~d} \boldsymbol{v}\left(Q^{\prime}\right)=\int_{P_{0}}^{P} \mathrm{~d} \boldsymbol{v}\left(Q^{\prime}\right)+\sum_{k=1}^{\infty} \boldsymbol{U}_{k}(P) \xi(Q)^{k}
$$

with $\xi(Q)$ being the local coordinate of the point $Q$ in the vicinity of the given point $P$, so that $\xi(P)=0$. The quantities $\omega_{m n}(P)$ define the holomorphic part of the expansion of the fundamental second kind differential $\omega(Q, S)$ near the point $P$ according to (2.7).

We restrict ourselves to the case of algebraic curves with a branch point at infinity, and take $P=(\infty, \infty)$ to be the base point where we expand all our functions. The winding vectors are in this case

$$
\boldsymbol{U}_{k}(\infty) \equiv \boldsymbol{U}_{k}=\mathcal{A}^{-1} \boldsymbol{R}_{k}, \quad k=1, \ldots, g,
$$

where $\boldsymbol{R}_{1}, \boldsymbol{R}_{2}, \ldots$ are residues of canonical holomorphic integrals multiplied by differentials of the second kind with poles of order $k$ at infinity, giving:

$$
\boldsymbol{R}_{k}=\left.\frac{1}{k} \frac{\mathrm{d}^{k-1}}{\mathrm{~d} \xi(Q)^{k-1}} \mathrm{~d} \boldsymbol{u}(Q) \frac{1}{\mathrm{~d} \xi(Q)}\right|_{Q=\infty} .
$$

Using the above definitions, we can see that the algebro-geometric $\tau$-function is given by the formula, equivalent to (1.8):

$$
\frac{\tau(\boldsymbol{t} ; \boldsymbol{u})}{\tau(\mathbf{0} ; \boldsymbol{u})}=\frac{\sigma\left(\sum_{k=1}^{\infty} \boldsymbol{R}_{k} t_{k}+\boldsymbol{u}\right)}{\sigma(\boldsymbol{u})} \exp \left\{\frac{1}{2} \sum_{k, l=0}^{\infty} \omega_{k, l}^{\mathrm{alg}} t_{k} t_{l}\right\} .
$$

In (3.4), $\omega_{k, l}^{\text {alg }}$ is the algebraic part of the holomorphic part $\omega_{k, l}$ of the expansion of the bi-differential as defined in (2.13). One can see that the non-algebraic part, i.e. the normalizing bi-linear form, is absorbed into the $\sigma$-function.

Once we have an expression involving derivatives of the sigma function, we need to convert this to an expression involving derivatives of the $\wp$-function. To do this we start with the definition of the $\zeta$ function (1.9), which we write in the form

$$
\sigma_{i}(\boldsymbol{u})=\zeta_{i}(\boldsymbol{u}) \sigma(\boldsymbol{u}), \quad i=1, \ldots, g
$$

then repeated differentiation gives us a ladder of relations which enable us to recursively express any derivative of $\sigma$ in terms of $\wp_{i j \ldots k}, \zeta_{i}$ and $\sigma$, all evaluated at $\mathbf{u}$.

$$
\begin{aligned}
\sigma_{i j}(\boldsymbol{u}) & =\sigma_{j}(\boldsymbol{u}) \zeta_{i}(\boldsymbol{u})-\sigma(\boldsymbol{u}) \wp_{i j}(\boldsymbol{u}) \\
\sigma_{i j k}(\boldsymbol{u}) & =\sigma_{j k}(\boldsymbol{u}) \zeta_{i}(\boldsymbol{u})-\sigma(\boldsymbol{u}) \wp_{i j k}(\boldsymbol{u})-\sigma_{k}(\boldsymbol{u}) \wp_{i j}(\boldsymbol{u})-\sigma_{j}(\boldsymbol{u}) \wp_{i k}(\boldsymbol{u}), \\
\text { etc } \ldots &
\end{aligned}
$$


In the following sections, we will look at different methods for constructing such relations between the derivatives of $\sigma$, and hence between the Abelian functions associated with the curve.

\section{The "ClASSICAL" METHOD}

The starting point for this approach is the Klein formula, which compares two different expressions for the fundamental bi-differential:

Theorem 4.1. Let the canonical holomorphic differentials of the curve $f(x, y)=$ 0 be represented in the form

$$
\mathrm{d} u_{i}(x, y)=\frac{\mathcal{U}_{i}(x, y)}{f_{y}(x, y)} \mathrm{d} x, \quad i=1, \ldots, g,
$$

where $\mathcal{U}_{k}(x, y)$ are monomials of their variables. Then the following g relations hold

$$
\begin{aligned}
& \sum_{i, j=1}^{g} \wp_{i j}\left(\int_{P_{0}}^{(x, y)} \mathrm{d} \boldsymbol{u}-\sum_{k=1}^{g} \int_{P_{0}}^{\left(x_{k}, y_{k}\right)} \mathrm{d} \boldsymbol{u}+\boldsymbol{K}_{P_{0}}\right) \mathcal{U}_{i}(x, y) \mathcal{U}_{j}\left(x_{k}, y_{k}\right) \\
& =\frac{\mathcal{F}\left(x, y ; x_{k}, y_{k}\right)}{\left(x-x_{k}\right)^{2}}, \quad k=1, \ldots, g .
\end{aligned}
$$

with polynomial $\mathcal{F}(P, Q)=\mathcal{F}(x, y ; z, w)$ defined in (2.12), an arbitrary base point of the Abel map $P_{0}$, and the corresponding vector of Riemann constants, $\boldsymbol{K}_{P_{0}}$.

As an application of this theorem consider the case of a curve with branch point at $P_{0}=(\infty, \infty)$. Let us tend $P=(x, y) \rightarrow(\infty, \infty)$. Both, right and left had sides of (4.1) have poles at infinity. Equating principal part of poles, we get a set of relations between $P_{k}=\left(x_{k}, y_{k}\right)$ and the multi-indexed $\wp$-symbols which can be interpreted as differential equations for the $\wp_{k, l}$-functions. In this way, the solution of the Jacobi inversion problem can also be derived in terms of $\wp$-functions.

4.1. Example: Hyperelliptic curve of genus two. The $\sigma$-functional realization of hyperelliptic functions of a genus two curve has already been discussed in many places, see e.g. [1, 3]. But we shall briefly describe here the principal points of the construction to convey the structure of the theory that we wish to develop for higher genera curves.

We consider the genus two hyperelliptic curve of example I. The algebraic part of the fundamental bi-differential is given as above in eqn. (2.14) [1].

Following the procedure in those papers, we equate principal parts of the highest (second order) pole to obtain

$$
\wp_{12}+x \wp_{11}-x^{2}=0
$$


which is the $x$-part of the Jacobi inversion problem for this curve. Then equating principal parts of the next, lower pole and using (4.2) leads to the relation

$$
y_{k}=-\wp_{112}-x_{k} \wp_{111}
$$

which completes the solution of the Jacobi inversion problem.

We can use these relations to eliminate $y$ and quadratic and higher terms in $x$, and we get from the next term

$$
\begin{aligned}
& \left(\frac{1}{2} \wp_{1111}-3 \wp_{11}^{2}-\frac{1}{2} \alpha_{4} \wp_{11}-2 \wp_{12}-\frac{1}{4} \alpha_{3}\right) x_{k} \\
& \quad+\frac{1}{2} \wp_{1112}-\frac{1}{2} \alpha_{4} \wp_{12}-3 \wp_{11} \wp_{12}+\wp_{22}=0 .
\end{aligned}
$$

This equation holds for both $x_{k}$, so the coefficients of different powers of $x_{k}$ must be identically zero, and solving for $\wp_{1111}$ and $\wp_{1112}$ we find

$$
\begin{aligned}
& \wp_{1111}=6 \wp_{11}^{2}+\alpha_{4} \wp_{11}+4 \wp_{12}+\frac{1}{2} \alpha_{3}, \\
& \wp_{1112}=6 \wp_{11} \wp_{12}+\alpha_{4} \wp_{12}-2 \wp_{22},
\end{aligned}
$$

the first two 4-index relations in the genus 2 case. A relation for 3 -index functions $\wp_{i j k}$ can be obtained by cross-derivation of (4.4) and (4.5):

$$
\wp_{122}+\wp_{11} \wp_{112}-\wp_{12} \wp_{111}=0 .
$$

Other equations are found from higher order terms. At every stage we need to substitute for higher derivative terms (such as $\wp_{11111}$, for example) by using derivatives of previously derived relations. In addition, multiplication by a 3 -index $\wp_{i j k}$ is sometimes useful, followed by substitution of known relations which are quadratic in the $\wp_{i j k}$. The first such relation is found to be

$$
\mathrm{Jac}_{6}: \quad \wp_{111}^{2}=4 \wp_{11}^{3}+\alpha_{3} \wp_{11}+\alpha_{4} \wp_{11}^{2}+4 \wp_{12} \wp_{11}+\alpha_{2}+4 \wp_{22} .
$$

This is one of the relations that describe the Jacobi variety of the curve as algebraic variety. These relations are well documented in [2], see also [3]. Below we show an alternative way to derive these relations.

We remark briefly that one variation on the classical method is to invoke the use of the sigma function expansion in the $u_{i}$ variables. The first few terms in the sigma expansion are derived from the lowest order expansion terms of (4.1). These can then be used in a bootstrap fashion to derive higher order PDEs for the $\wp$ functions. This approach was essential in the genus six $(4,5)$ case considered in [10], which is discussed in more detail in that paper.

\section{Derivation of integrable hierarchies via PlüCker Relations}

The key to this approach is Sato's formula, Theorem (5.1) below. This gives an expansion of a ratio of $\tau$-functions, $\tau(\boldsymbol{t} ; \boldsymbol{u}) / \tau(\mathbf{0} ; \boldsymbol{u})$, as a series of rational expressions in the $\tau$-function and its derivatives at $\boldsymbol{t}=0$. The $\boldsymbol{t}$-dependence is given in terms of Schur polynomials $s_{\lambda}(\boldsymbol{t})$ in the times $t_{i}$. The coefficients of these polynomials are determinants of differential expressions of $\tau$, which are 
Plücker coordinates on a Grassmannian. Such coordinates satisfy the Plücker relations - each partition $\lambda$ can be expanded in hooks, and the corresponding Plücker coordinates are expressible, analogously to Giambelli's formula, as determinants of single hook partitions. These relations give the differential relations for the Abelian functions which we seek.

For any partition $\lambda: \alpha_{1} \geq \alpha_{2} \geq \ldots \geq \alpha_{n}$ of $|\lambda|=\sum_{i=1}^{n} \alpha_{i}$, the Schur polynomial of $n$ variables $x_{1}, \ldots, x_{n}$ is defined by

$$
s_{\lambda}(\boldsymbol{x})=\operatorname{det}\left(p_{\alpha_{i}-i+j}(\boldsymbol{x})\right)_{i, j=1, \ldots, n},
$$

where the elementary Schur functions $p_{m}(\boldsymbol{x})$ are generated by the series,

$$
\sum_{m=0}^{\infty} p_{m}(\boldsymbol{x}) t^{m}=\exp \left\{\sum_{n=1}^{\infty} x_{n} t^{n}\right\}
$$

The first few Schur polynomials are

$s_{1}(\boldsymbol{x})=x_{1}$,

$s_{2}(\boldsymbol{x})=x_{2}+\frac{1}{2} x_{1}^{2}, \quad s_{1,1}(\boldsymbol{x})=-x_{2}+\frac{1}{2} x_{1}^{2}$,

$s_{3}(\boldsymbol{x})=x_{3}+x_{1} x_{2}+\frac{1}{6} x_{1}^{3}, \quad s_{2,1}(\boldsymbol{x})=-x_{3}+\frac{1}{3} x_{1}^{3}, \quad s_{1,1,1}(\boldsymbol{x})=x_{3}-x_{1} x_{2}+\frac{1}{6} x_{1}^{3}$

$s_{4}(\boldsymbol{x})=x_{4}+x_{1} x_{3}+\frac{1}{2} x_{2}^{2}+\frac{1}{2} x_{1}^{2} x_{2}+\frac{1}{24} x_{1}^{4}$, etc.

The Cauchy-Littlewood formula

$$
\exp \left\{\sum_{n=1}^{\infty} n x_{n} y_{n}\right\}=\sum_{\lambda} s_{\lambda}(\boldsymbol{x}) s_{\lambda}(\boldsymbol{y})
$$

where $s_{\lambda}(\boldsymbol{x})$ is the Schur function of the partition $\lambda: \alpha_{1} \geq \alpha_{2} \geq \ldots \geq \alpha_{n}$, leads to the Taylor expansion in the form

$$
f(\boldsymbol{x})=\left.\exp \left\{\sum_{n=1}^{\infty} x_{n} \frac{\partial}{\partial y_{n}}\right\} f(\boldsymbol{y})\right|_{\boldsymbol{y}=0}=\left.\sum_{\lambda} s_{\lambda}(\boldsymbol{x}) s_{\lambda}\left(\frac{1}{n} \frac{\partial}{\partial y_{n}}\right) f(\boldsymbol{y})\right|_{\boldsymbol{y}=0}
$$

We now introduce the Frobenius notation for partitions. Single hook partitions $(\alpha, \beta)$ are denoted $\left(\alpha+1,1^{\beta}\right)$. All partitions can be decomposed into finitely many hooks:

$$
(\lambda)=\left(\alpha_{1}, \ldots, \alpha_{r} \mid \beta_{1}, \ldots, \beta_{r}\right) .
$$

The total number of hooks $r$ is called the rank of the partition $\lambda$, [27]. In particular, a partition which decomposes into two hooks (rank 2) is written as:

$$
\left(n, m, 2^{k}, 1^{l}\right)=(n-1, m-2 \mid k+l+1, k),
$$

where $n>m>1, k \geq 0$ and $l \geq 0$. Most of the formulae below are derived using rank 2 partitions. 
Giambelli's formula now shows how to expand a Schur function $s_{\lambda}$ in hooks,

$$
s_{\lambda}(\boldsymbol{x})=\operatorname{det}\left(s_{\left(\alpha_{i}, \beta_{j}\right)}(\boldsymbol{x})\right)_{1 \leq i, j \leq r},
$$

Theorem 5.1 (Sato formula ). Let $\tau(\boldsymbol{t} ; \boldsymbol{u})$ be any function of vector arguments

$$
\boldsymbol{U}_{1} t_{1}, \boldsymbol{U}_{2} t_{2}, \ldots, \quad \boldsymbol{t}=\left(t_{1}, t_{2}, \ldots\right) \in \mathbb{C}^{\infty}
$$

where $\boldsymbol{U}_{1}, \boldsymbol{U}_{2} \ldots$ is an infinite set of constant complex vectors from $\mathbb{C}^{g}$ and $\boldsymbol{u} \in \mathbb{C}^{g}$ is a parameter. Suppose that $\tau(\mathbf{0} ; \boldsymbol{u}) \neq 0$. Then for any partition $(\lambda)=\left(\alpha_{1}, \ldots, \alpha_{r} \mid \beta_{1}, \ldots, \beta_{r}\right)$ and any $\boldsymbol{u} \in \mathbb{C}^{g}$

$$
\frac{\tau(\boldsymbol{t} ; \boldsymbol{u})}{\tau(\mathbf{0} ; \boldsymbol{u})}=\sum_{\lambda} s_{\lambda}(\boldsymbol{t}) \operatorname{det}\left((-1)^{\beta_{j}+1} A_{\left(\alpha_{i} \mid \beta_{j}\right)}(\boldsymbol{u})\right)
$$

where the $A_{(m \mid n)}(\boldsymbol{u})$ with $m \geq 0, n \geq 0$ form a linear basis of the Grassmannian:

$$
\begin{aligned}
A_{(m \mid n)}(\boldsymbol{u}) & =-A_{(n \mid m)}(-\boldsymbol{u}) \\
& =\left.(-1)^{n+1} s_{m+1,1^{n}}\left(\partial_{\boldsymbol{t}}\right) \tau(\boldsymbol{t} ; \boldsymbol{u})\right|_{\boldsymbol{t}=0} \tau(\mathbf{0} ; \boldsymbol{u})^{-1} \\
& =\left.\sum_{\alpha=0}^{m} p_{n+\alpha+1}\left(-\partial_{\boldsymbol{t}}\right) p_{m-\alpha}\left(\partial_{\boldsymbol{t}}\right) \tau(\boldsymbol{t} ; \boldsymbol{u})\right|_{\boldsymbol{t}=0} \tau(\mathbf{0} ; \boldsymbol{u})^{-1}
\end{aligned}
$$

and

$$
\partial_{t}=\left(\frac{\partial}{\partial t_{1}}, \frac{1}{2} \frac{\partial}{\partial t_{2}}, \frac{1}{3} \frac{\partial}{\partial t_{3}}, \ldots,\right) .
$$

Theorem 5.2 (Plücker relations). For any partition $(\lambda)=\left(\alpha_{1}, \ldots, \alpha_{r} \mid \beta_{1}, \ldots, \beta_{r}\right)$ and any $\boldsymbol{u} \in \mathbb{C}^{g}$, the $\tau$-function satisfies:

$$
\left.\tau(\mathbf{0} ; \boldsymbol{u})^{r-1} s_{\lambda}\left(\partial_{\boldsymbol{t}}\right) \tau(\boldsymbol{t} ; \boldsymbol{u})\right|_{\boldsymbol{t}=0}=\operatorname{det}\left(\left.s_{\left(\alpha_{i} \mid \beta_{j}\right)}\left(\partial_{\boldsymbol{t}}\right) \tau(\boldsymbol{t} ; \boldsymbol{u})\right|_{\boldsymbol{t}=0}\right) \text {. }
$$

In particular, for any curve $X$, its Sato algebro-geometric $\tau$-function has the expansion

$$
\begin{aligned}
\frac{\tau(\boldsymbol{t} ; \boldsymbol{u})}{\tau(\mathbf{0} ; \boldsymbol{u})} & =1+A_{(0 \mid 0)}(\boldsymbol{u}) s_{1}(\boldsymbol{t})+A_{(1 \mid 0)}(\boldsymbol{u}) s_{2}(\boldsymbol{t}) \\
& +A_{(0 \mid 1)}(\boldsymbol{u}) s_{1,1}(\boldsymbol{t})+\ldots \\
& =1+A_{(0 \mid 0)} t_{1}+A_{(1 \mid 0)}\left(t_{2}+\frac{1}{2} t_{1}^{2}\right)+A_{(0 \mid 1)}(\boldsymbol{u})\left(-t_{2}+\frac{1}{2} t_{1}^{2}\right)+\ldots
\end{aligned}
$$

where $A_{(m \mid n)}(\boldsymbol{u})$ as defined in (5.2) are the elements of the semi-infinite matrix A,

$$
A=\left(\begin{array}{cccc}
A_{(0 \mid 0)}(\boldsymbol{u}) & A_{(0 \mid 1)}(\boldsymbol{u}) & A_{(0 \mid 2)}(\boldsymbol{u}) & \ldots \ldots \\
A_{(1 \mid 0)}(\boldsymbol{u}) & A_{(1 \mid 1)}(\boldsymbol{u}) & A_{(1 \mid 2)}(\boldsymbol{u}) & \ldots \ldots \\
A_{(2 \mid 0)}(\boldsymbol{u}) & A_{(2 \mid 1)}(\boldsymbol{u}) & A_{(2 \mid 2)}(\boldsymbol{u}) & \ldots \ldots \\
\vdots & \vdots & \vdots & \ldots
\end{array}\right)=\left(\boldsymbol{A}_{0}, \boldsymbol{A}_{1}, \ldots \ldots\right)
$$


with infinite vectors $\boldsymbol{A}_{i}, i=1,2, \ldots$ To more complicated partitions such as $\left(m_{1}, \ldots, m_{k} \mid n_{1}, \ldots, n_{k}\right)$ we associate according to the Giambelli formula certain minors of $A$,

$$
A_{\left(m_{1}, \ldots, m_{k} \mid n_{1}, \ldots, n_{k}\right)}(\boldsymbol{u})=\operatorname{det}\left(A_{\left(m_{i} \mid n_{j}\right)(\boldsymbol{u})}\right)_{i, j=1 \ldots, k} .
$$

Note that these $A$ 's are not independent - they satisfy the Plücker relations. These are a family of differential equations satisfied by $\tau$, that represent a completely integrable hierarchy of KP-type.

The definitions and relations given above are valid for any multivariate function $\tau(\boldsymbol{t} ; \boldsymbol{u})$. Below we consider functions $\tau$-functions constructed on the Jacobi varieties of algebraic curves.

To each symbol we shall put in correspondence its weight

$$
\wp_{\underbrace{}_{k_{1}}}^{1, \ldots, 1}, \underbrace{2, \ldots, 2}_{k_{2}}, \ldots, \underbrace{g, \ldots, g}_{k_{g}} \Leftrightarrow \sum_{j=1}^{g} k_{j} w_{j},
$$

where $w_{i}$ is the order of vanishing of the holomorphic integral $\int \mathrm{d} u_{i}$ at infinity. In other words, if the curve has a Weierstrass point at infinity, then $w_{i}=1<$ $w_{2}<\ldots<w_{g}$ is the Weierstrass gap sequence at infinity.

For a given weight $W$ consider all Young diagrams which decompose into only two hooks, and write corresponding relations between multi-index symbols $\wp_{i_{1}, \ldots, i_{n}}$. The first non-trivial Young diagram corresponds to the partition $\lambda=$ $(2,2)$. There is only one multi-index symbol of weight 4 , that is $\wp_{1111}(\boldsymbol{u})$ and its corresponding Plücker relation for any curve is of the form

$$
\wp_{1111}(\boldsymbol{u})=\text { polynomial of even symbols } \wp_{i j}(\boldsymbol{u})
$$

For higher weights a larger number of multi-index functions can be constructed, and this number grows rapidly with increasing weight. To find them in terms in the form of polynomials of two-index functions, we shall write Plücker relations corresponding to independent diagrams of the same weight, and solve the corresponding linear systems. The technique is best illustrated by examples.

We consider the genus two hyperelliptic curve, with the differentials and the bi-differential chosen as in example I above. The simplest class of non-trivial Plücker relations is found from the class of Young diagrams which may be decomposed into two hooks, that is, those of the form $\left(2+m, 2+n, 2^{k}, 1^{l}\right)$, with $m \geq n \geq 0, k \geq 0, l \geq 0$. In Frobenius 'hook' notation these are $(m+$ $1, n \mid k+l+1, l)$. The corresponding Plücker relations read

$$
A_{(m+1, n \mid k+l+1, l)}=\left|\begin{array}{cc}
A_{(m+1, k+l+1)} & A_{(m+1, l)} \\
A_{(n, k+l+1)} & A_{(n, l)}
\end{array}\right| .
$$

These equations are all bilinear partial differential equations in the $\tau$-function. They may be expanded in terms of the Kleinian $\wp_{i j}$ and $\zeta_{i}$ functions. 
The first Young diagram leading to a non-trivial Plücker relation corresponds to the partition $\lambda=(2,2)$, with Young diagram

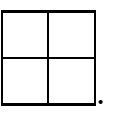

Writing (5.3) in this case we obtain after simplification

$$
\mathrm{KdV}_{4}: \quad \wp_{1111}(\boldsymbol{u})=6 \wp_{11}^{2}(\boldsymbol{u})+4 \wp_{12}(\boldsymbol{u})+\alpha_{4} \wp_{11}(\boldsymbol{u})+\frac{1}{2} \alpha_{3} .
$$

This is of course the same equation as (4.4). The weight of the diagram is 4 in this case, which is the same as the weight of the equation, defined as the weighted sum of indices in which the index " 1 " has weight 1 and index "2" has weight 3 . We will use this weight correspondence in other cases too, and will denote the weight of the object by subscript $i+3 j$ where $i$ and $j$ are respectively the numbers of 1's and 2's in the multi-index relation. The relation (5.7) is the analogue of Weierstrass' equation for $\wp^{\prime \prime}$ in the genus 1 case.

The next group of diagrams are of weight 5 , and correspond to the partitions $\lambda=(3,2)$ and $\lambda=(2,2,1)$, with their transposes, which give the same equations, in the hyperelliptic case. Both these Plücker relations lead to the equation

$$
\left(\zeta_{1}(\boldsymbol{u})+\frac{\partial}{\partial u_{1}}\right) \mathrm{KdV}_{4}=0
$$

i.e. the Young diagram of weight 5 gives no new equations and we conclude that the following correspondence is valid

$$
\left\{\begin{array}{c}
\lambda=(2,2) \\
\lambda=(3,3), \quad \lambda=(2,2,1)
\end{array}\right\} \Longleftrightarrow \mathrm{KdV}_{4}
$$

At weight 6 we have three independent Young diagrams with $(2,2)$ centres
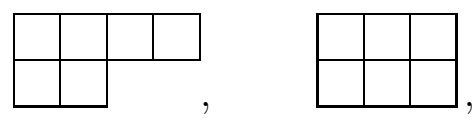

and

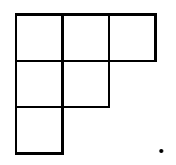

The other two diagrams of weight 6

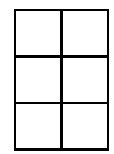

and

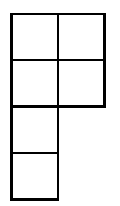

again give the same results as their transposes.

Remark 5.3. The Plücker relations associated with any Young diagram and its transpose are always the same for a hyperelliptic curve; however for general curves, this is no longer true. 
These three independent diagrams give an overdetermined system of three equations. After substituting for higher derivatives of the known relation (5.7), we can solve for the two unknowns $\wp_{1112}$ and $\wp_{111}^{2}$ to get

$$
\begin{aligned}
\mathrm{KdV}_{6}: & \wp_{1112}=6 \wp_{12} \wp_{11}-2 \wp_{22}+\alpha_{4} \wp_{12} \\
\operatorname{Jac}_{6}: & \wp_{111}^{2}=4 \wp_{11}^{3}+\alpha_{3} \wp_{11}+\alpha_{4} \wp_{11}^{2}+4 \wp_{12} \wp_{11}+\alpha_{2}+4 \wp_{22}
\end{aligned}
$$

The relation (5.8) is another generalization of the equation for $\wp^{\prime \prime}$ in the genus one case, and is identical to (4.5) found using the classical method. The relation (5.9) corresponds to Weierstrass' equation for $\left(\wp^{\prime}\right)^{2}$ in the genus one case. In what follows we will always assume that higher derivatives of the known relations at a lower weight have been eliminated.

At weight 7 we have 5 independent Young diagrams with $(2,2)$ centres. The resulting overdetermined system of equations, of weight 7 , contain $\zeta_{1}$ multiplied by linear combinations of the two weight 6 relations (5.9) get a relation at weight 7 , namely

$$
\wp_{12} \wp_{111}-\wp_{122}-\wp_{112} \wp_{11}=0,
$$

as noted above. We refer to relations of this type, linear in the three-index $\wp_{i j k}$, as quasilinear. These have no counterpart in the genus 1 theory. They can also be derived by cross-differentiation between the two relations (5.7,5.8), since

$$
\frac{\partial}{\partial u_{2}} \wp_{1111}=\frac{\partial}{\partial u_{1}} \wp_{1112}
$$

Equations $K d V_{4}$ and $K d V_{6}$ describe the genus 2 solutions of the KdV hierarchy associated with the given curve. To describe the Jacobi variety and the Kummer variety we must consider diagrams of higher weights.

For the diagram of weight 8 we find a set of eight overdetermined equations with solution given by

$$
\begin{aligned}
\mathrm{Jac}_{8}: \wp_{112}^{2} & =\alpha_{0}-4 \wp_{22} \wp_{12}+\alpha_{4} \wp_{12}^{2}+4 \wp_{11} \wp_{12}^{2} \\
\mathrm{KdV}_{8}: \wp_{1122} & =2 \wp_{11} \wp_{22}+4 \wp_{12}^{2}+\frac{1}{2} \alpha_{3} \wp_{12},
\end{aligned}
$$

And at weight 9 the only new relation is the quasilinear relation

$$
8 \wp_{122} \wp_{11}-4 \wp_{12} \wp_{112}-4 \wp_{222}+2 \alpha_{4} \wp_{122}-\alpha_{3} \wp_{112}-4 \wp_{111} \wp_{22}=0
$$

At weight 10 we have 18 overdetermined system of equations, which can be solved for the 3 functions of weight $10, \wp_{1222}, \wp_{112}^{2}, \wp_{111} \wp_{122}$, giving

$$
\begin{aligned}
\mathrm{KdV}_{10}: \wp_{1222} & =6 \wp_{12} \wp_{22}+\alpha_{2} \wp_{12}-\frac{1}{2} \alpha_{1} \wp_{11}-\alpha_{0} \\
\operatorname{Jac}_{10}^{(1)}: \wp_{111} \wp_{122} & =-\frac{1}{2} \alpha_{1} \wp_{11}+2 \wp_{22} \wp_{11}^{2}+2 \wp_{11} \wp_{12}^{2}+\alpha_{2} \wp_{12}+4 \wp_{22} \wp_{12}+\frac{1}{2} \alpha_{3} \wp_{12} \wp_{11} \\
\operatorname{Jac}_{10}^{(2)}: \wp_{112}^{2} & =\alpha_{0}-4 \wp_{22} \wp_{12}+\alpha_{4} \wp_{12}^{2}+4 \wp_{11} \wp_{12}^{2}
\end{aligned}
$$


The equations $\mathrm{Jac}_{8}, \mathrm{Jac}_{10}^{(1)}, \mathrm{Jac}_{10}^{(2)}$ represent an embedding of the Jacobi variety as a 3 -dimensional algebraic variety into the complex space $\mathbb{C}^{5}$ whose coordinates are $\wp_{11}, \wp_{12}, \wp_{22}, \wp_{111}, \wp_{112}$

At weight 12 we get the final 4-index relation for $\wp_{2222}$ and two quadratic 3 -index relations for $\wp_{112} \wp_{122}$ and $\wp_{111} \wp_{222}$. We can continue in this manner at weight 14 to get more quadratic 3 -index relations 3. At the odd weights $11,13,15$, we get quasilinear relations which can also be found by crossdifferentiation. As a practical point we note that the equations we derive can often contain ideals generated by the lower weight relations, and some work is required to identify genuinely new relations.

At weight 16 we have 117 independent relations giving an overdetermined system of equations (we have checked only a selection of these). At this weight a new feature occurs. As well as the equations expressing the quadratic 3index term $\wp_{122} \wp_{222}$ in terms of cubics in the $\wp_{i j}$, we have terms which are quartic in the $\wp_{i j}$. We can pick one of these quartic terms, say $\wp_{12}^{4}$, and solve for this and for $\wp_{122} \wp_{222}$ to give us two relations. The relation involving a quartic in the $\wp_{i j}$ is just the Kummer variety of the curve. This is the quotient of the Jacobi variety, $\operatorname{Kum}(X)=\operatorname{Jac}(X) /(\boldsymbol{u} \rightarrow-\boldsymbol{u})$. In the case $g=2$, the Kummer variety is a surface in $\mathbb{C}^{3}$ which is given analytically by a quartic equation. This relation can can also be found from the identity

$$
\left(\wp_{111}^{2}\right)\left(\wp_{112}^{2}\right)-\left(\wp_{111} \wp_{112}\right)^{2}=0 .
$$

The same quartic also appears, multiplied by various factors, at higher weights.

5.1. Example: Trigonal curve of genus three. As before we consider Example II, (2.3) whose holomorphic differentials are given in (2.x) [11, 5, 12]. Here we generally follow the notation of [12]). The fundamental second kind differential is (2.16), (2.17), (2.19).

The first Young diagram leading to a non-trivial Plücker relation, as in the genus 2 case, corresponds to the partition $\lambda=(2,2)$. Writing (5.3) in this case we obtain, after simplification,

$$
\wp_{1111}=6 \wp_{11}^{2}-3 \wp_{22} \text {. }
$$

The weight of the diagram is again 4 in this case, which is the same as the weight of the equation, defined as the weighted sum of indices in which the index " 1 " has weight 1 , index " 2 " has weight 2 , and index " 3 " has weight 5. We will use this weight correspondence in other cases too, and will denote the weight of the object by subscript $i+2 j+5 k$ where $i, j$ and $k$ are respectively the numbers of 1's, 2's and 3's in the multi-index relation.

In the trigonal case we no longer longer have the symmetry about the diagonal of the diagram that we have in the genus 2 case, but we can restrict ourselves by taking the symmetric or antisymmetric combination of the two 
diagrams related by transposition. In the weight 5 case we have the antisymmetric combination

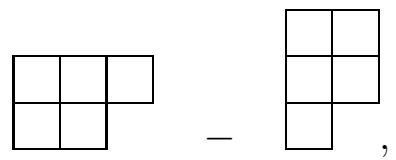

which gives the weight 5 trigonal PDE

$$
\wp_{1112}=6 \wp_{11} \wp_{12}+3 \mu_{3} \wp_{11} .
$$

For the symmetric case we get a derivative of the weight 4 equation, plus $\zeta_{1}$ multiplied by the same equation. With even (odd) weights, the symmetric (antisymmetric) combinations give the 4 -index $\wp_{i j k l}$ relations.

At weight 6 we have the three symmetric diagrams

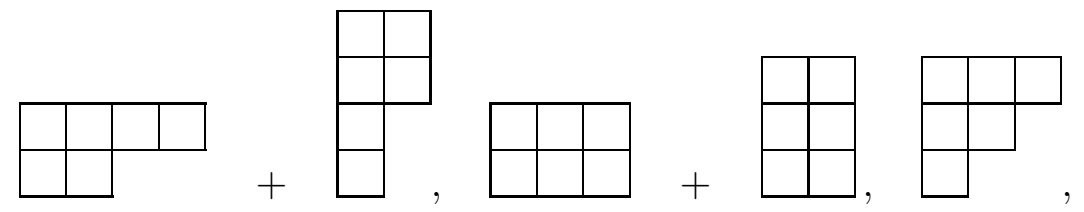

which give a set of three overdetermined equations with the unique solution

$$
\begin{aligned}
\wp_{111}^{2} & =4 \wp_{11}^{3}+\wp_{12}^{2}+4 \wp_{13}-4 \wp_{11} \wp_{22}, \\
\wp_{1122} & =4 \wp_{13}+2 \mu_{6}+4 \wp_{12}^{2}+2 \wp_{11} \wp_{22}+3 \mu_{3} \wp_{12} .
\end{aligned}
$$

Continuing in this way we recover the strictly trigonal versions of the full set of equations given in [12].

\section{Derivation of the integrable hierarchies Via RESidues}

This method of deriving integrable equations on the Jacobians of algebraic curves is based on the following relation due to Sato, [28], [29], as applied to the algebro-geometric context by Fay, [15].

The Hirota bilinear equations

$$
\begin{aligned}
\operatorname{Res}_{\xi=0} \frac{1}{\xi^{2}}\left[\exp \left\{\sum_{n=1}^{\infty} t_{n} \zeta^{-n}\right\} \exp \left\{-\sum_{n=1}^{\infty} \frac{\xi^{n}}{n} \frac{\partial}{\partial t_{n}}\right\} \tau(\boldsymbol{t} ; \boldsymbol{u})\right. \\
\left.\exp \left\{\sum_{n=1}^{\infty} t_{n}^{\prime} \xi^{-n}\right\} \exp \left\{-\sum_{n=1}^{\infty} \frac{\xi^{n}}{n} \frac{\partial}{\partial t_{n}^{\prime}}\right\} \tau\left(\boldsymbol{t}^{\prime} ;-\boldsymbol{u}\right)\right]=0,
\end{aligned}
$$

where $\xi=\xi(Q)$ is the local coordinate of the point $Q$ near $P, \xi(P)=0$ defining the KP hierarchy are equivalent to the following Bilinear Identity

Denote $\Omega(\boldsymbol{f})$ the differential

$$
\Omega(\boldsymbol{t}, P)=\sum_{k=1}^{\infty} k t_{k} \Omega_{k}(P)
$$


where $\Omega_{k}(P)$ are normalized second kind differentials with $k$-th order poles at infinity, so that $\Omega(\boldsymbol{t}, P)$ has an essential singularity

$$
\Omega(\boldsymbol{t}, P) \simeq\left(\sum_{n=1}^{\infty} n t_{n} \xi^{-n-1}+O(1)\right) d \xi
$$

and the $\Omega_{k}(P)$ are defined by

$$
\begin{array}{r}
\Omega_{k}(P)=\frac{\mathrm{d} \xi}{\xi^{k+1}}-\sum_{m=1}^{\infty} \frac{1}{m} \omega_{k m} \xi^{m-1} \mathrm{~d} \xi \\
\oint_{\mathfrak{a}_{j}} \Omega_{k}(P)=0, j=1, \ldots, g .
\end{array}
$$

Theorem 6.1 (Bilinear Identity). Let $S^{1}$ be a small circle about the point $p \in X$, then

$$
\begin{gathered}
\int_{S^{1}} \theta\left(\mathfrak{A}(Q)-\mathfrak{A}(P)-\sum_{i=1}^{\infty} \boldsymbol{U}_{i} t_{i}-\boldsymbol{u}\right) \theta\left(\mathfrak{A}(Q)-\mathfrak{A}(P)-\sum_{i=1}^{\infty} \boldsymbol{U}_{i} t_{i}^{\prime}+\boldsymbol{u}\right) \\
\times \exp \left\{\int_{P_{0}}^{P} \Omega\left(\boldsymbol{t}+\boldsymbol{t}^{\prime}, P\right)\right\} E^{-2}(P, Q) \mathrm{d} \xi(Q)=0,
\end{gathered}
$$

where $\mathfrak{A}(P)=\int_{\infty}^{P} \mathrm{~d} \boldsymbol{v}$ - Abelian image of a point $P, \xi(Q)$ is the local coordinate of the point $Q$ near $P, \xi(P)=0, E(P, Q)$ is the Schottky-Klein prime form (2.9) and the integration is around the unit circle centred at the point $p, S^{1}=$ $\{q,|\zeta(q)|=1\}$.

Proof. Note that the integrand in (6.4) is holomorphic in $q$ in the interior of $S^{1}$ except at its centre $Q=P$ where it has an isolated essential singularity with zero residue. Therefore this integral vanishes.

To prove the equivalence of (6.1) and (6.4) note first that

$$
\Omega\left(\boldsymbol{t}+\boldsymbol{t}^{\prime}\right)=\Omega(\boldsymbol{t})+\Omega\left(\boldsymbol{t}^{\prime}\right) .
$$

In (6.4), the factor

$$
\theta\left(\mathcal{A}(Q)-\mathcal{A}(P)-\sum_{i=1}^{\infty} \boldsymbol{U}_{i} t_{i}-\boldsymbol{u}\right) \exp \left\{\int_{P_{0}}^{P} \Omega(\boldsymbol{t})\right\}
$$

can be expressed as

$$
\exp \left\{\sum_{n=1}^{\infty} t_{n} \xi^{-n}\right\} \theta\left(\mathcal{A}(Q)-\mathcal{A}(P)-\sum_{i=1}^{\infty} \boldsymbol{U}_{i} t_{i}-\boldsymbol{u}\right) \exp \left\{\sum_{m, n=1}^{\infty} \omega_{m n} t_{n} \frac{\xi^{m}}{n}\right\} .
$$


Taking into account the form (3.2) for $\tau(\boldsymbol{t} ; \boldsymbol{u})$, the last two factors in the above formula may be expressed

$$
\theta\left(\mathcal{A}(Q)-\mathcal{A}(P)-\sum_{i=1}^{\infty} \boldsymbol{U}_{i} t_{i}-\boldsymbol{u}\right) \exp \left\{\sum_{m, n=1}^{\infty} \omega_{m n} t_{n} \frac{\xi^{m}}{n}\right\}=\exp \left\{-\sum_{n=1}^{\infty} \frac{\xi^{n}}{n} \frac{\partial^{n}}{\partial t_{n}}\right\} \tau(\boldsymbol{t} ; \boldsymbol{u}),
$$

which completes the proof of the equivalence of (6.4) and (6.1).

Remark 6.2. Equations of this form were first written down in terms of 'vertex operators'

$$
\exp \left\{\sum_{1}^{\infty} t_{n} x^{-n}\right\} \exp \left\{-\sum_{1}^{\infty} \frac{x^{n}}{n} \frac{\partial}{\partial t_{n}}\right\}
$$

for general $\tau$-functions in the work of Sato. They may be understood as generating functions for integrable hierarchies of Hirota bilinear equations.

Using this theorem we may obtain partial differential equations relating the Kleinian symbols, $\wp_{i j}, \wp_{i j k}$ etc. To do this we now substitute the $\tau$-function (1.8) into this expression and compute the residue. This is parameterized by $\boldsymbol{t}$ and $\boldsymbol{e}$. The coefficients of monomials in $\boldsymbol{t}$ are differential-difference expressions in $\sigma$ at arguments $+\boldsymbol{u}$ and $\boldsymbol{- u}$. To get purely differential expressions, we now let $\boldsymbol{u} \rightarrow 0$; the 'time' derivatives act on $\sigma$ via its $\boldsymbol{u}$-dependence, though, so $\sigma_{i}(\boldsymbol{u})=-\sigma_{i}(-\boldsymbol{u}) \rightarrow \pm \sigma_{i}(0)$, etc. We then replace the derivatives of the $\sigma$-function by derivatives of the $\wp$ function using the recursive relations (3.5) described earlier. This recovers the partial differential equations for the Kleinian functions which we need.

\section{Example I}

In the genus 2 hyperelliptic case, the first nonvanishing relations occur at $t$-weight 3 in the $t_{i}$, i.e. the coefficients of $t_{1}^{3}$ or $t_{3}$. Both these give the first 4-index relation

$$
\wp_{1111}=6 \wp_{11}^{2}+\alpha_{4} \wp_{11}+4 \wp_{12}+\frac{1}{2} \alpha_{3} .
$$

The next non-zero term is at weight 5 , where we recover the relation for $\wp_{1112}$, etc. In contrast with the previous section, this approach only gives the even derivative relations at odd $t$-weights.

The calculations by this approach, because they involve bilinear products of $\tau$-functions, soon become very computer-intensive, and we have not pursued them very far.

Example II Within this method we are able to recover all quadratic relations for four indexed symbols $\wp_{i j k l}, 1 \geq i, j, k, l \leq 3$ and cubic relations for three indexed symbols $\wp_{i j k}^{2}$. We report here the quartic relation between even variables, which must be one of the relations defining the Kummer variety of the (3,4)-curve,

$$
\wp_{12}^{4}-\wp_{22}^{3}-2 \wp_{11} \wp_{33}+2 \wp_{13}^{2}+4 \wp_{12}^{2} \wp_{13}+\wp_{1113} \wp_{22}-6 \wp_{11} \wp_{13} \wp_{22}+4 \wp_{11} \wp_{12} \wp_{23}
$$




$$
\begin{aligned}
& +\wp_{11}^{2} \wp_{22}^{2}-2 \wp_{11} \wp_{12}^{2} \wp_{22}-\frac{4}{3} \wp_{1113} \wp_{11}^{2}+8 \wp_{11}^{3} \wp_{13}+2 \mu_{12}+4 \mu_{6} \wp_{11}^{3}-4 \mu_{6} \wp_{11} \wp_{22} \\
& +3 \mu_{3} \wp_{12} \wp_{13}+3 \mu_{3} \wp_{11} \wp_{23}+\mu_{3} \wp_{12}^{3}+2 \mu_{6} \wp_{13}+\mu_{6} \wp_{12}^{2}+\mu_{9} \wp_{12}-\mu_{3}^{2} \wp_{11}^{3} \\
& -3 \mu_{3} \wp_{11} \wp_{12} \wp_{22}=0 .
\end{aligned}
$$

This relation is of independent interest since it is of weight 12 and cannot be written in the form (5.10). The detailed structure of the Kummer variety in the $(3,4)$ case is receiving further investigation and will be reported on elsewhere.

\section{Discussion}

In view of the "compare and contrast" objectives of our paper, the reader may wish to know which of these methods is the most effective when calculating the required PDEs. Unfortunately this is not an easy question to answer. Currently the two methods associated with the $\tau$-function take rather longer to execute, with the residue method in particular being slow and with large memory overheads. However this may be due in part to the fact that we have extensive experience over ten years or more in developing computer algebra code for the "Classical method" in all its variations. We have much less experience in working with the tau-function methods. So it may be that with more study and with more computational experience, the $\tau$-function methods become more competitive. We would stress, however, that both $\tau$-function methods are more systematic than the classical methods, and may provide a suitable way of calculating the PDEs associated with more complicated curves.

Acknowledgments The work was partially supported by the European Science Foundation Programme MISGAM (Methods of Integrable Systems, Geometry and Applied Mathematics), the Imperial College (London) section of

the ENIGMA network, and a Fellowship (VZE) in the Hanse-Wissenschaftskolleg in Delmenhorst in 2010, where the final version of the paper was prepared. The authors are grateful to L. Haine for discussions and for bringing to our attention the useful unpublished manuscript by J. Fay, [15]. VZE would like to thank John Harnad for many discussions and inspiring ideas, as well as A. Nakayashiki for sending his paper on a closely related subject before publication. Some of this work was carried out whilst JCE and VZE were visiting Iwate University, and these authors are grateful to Y. Onishi for hospitality and for financial support from JSPS grant 19540002.

\section{REFERENCES}

[1] H. F. Baker, Abel's theorem and the allied theory of theta functions, Cambridge Univ. Press, Cambridge, 1897, Reprinted in 1995.

[2] H. F. Baker, Multiply Periodic Functions, Cambridge Univ. Press, Cambridge, 1907. 
[3] V. M. Buchstaber, V. Z. Enolskii, and D. V. Leykin, Kleinian functions, hyperelliptic Jacobians and applications, Reviews in Mathematics and Mathematical Physics (London) (S. P. Novikov and I. M. Krichever, eds.), vol. 10:2, Gordon and Breach, 1997, pp. $1-125$.

[4] V. M. Buchstaber, V. Z. Enolskii, and D. V. Leykin, $\sigma$-functions of $(n, s)$-curves, Uspekhi Matem. Nauk 54 (1999), no. 3, 155-156.

[5] V. M. Buchstaber, V. Z. Enolskii, and D. V. Leykin, Uniformisation of Jacobi varieties of trigonal curves and nonlinear differential equations, Func. Anal. Appl. 34 (2000), no. $3,159-171$.

[6] V. M. Buchstaber and D. V. Leykin, Addition Laws on Jacobian Variety of Plane Algebraic C, Proceedings of the Steklov Institute of Mathematics 251 (2005), 1-72.

[7] V. M. Buchstaber and D. V. Leykin, Solution of the problem of differentiation of Abelian functions over parameters for families of $(n, s)$ curves, Functional. Anal. and its Appl. 42 (2008), no. 4, 268-278, Translation of: Funksional. Anal. i Prilozhen, 42(2008) 24-36.

[8] E. Date, M. Jimbo, M. Kashiwara, and T. Miwa, Transformation groups for soliton equations, Proc. RIMS Symposium on Nonlinear Integrable Systems - Classical and Quantum Field Theory (M. Jimbo and T. Miwa, eds.), World Scientific, 1983.

[9] B. A. Dubrovin, V. B. Matveev, and S. P. Novikov, Nonlinear equations of the KdV type, finite gap linear operators and abelian varieties, Uspekhi Matem. Nauk 31 (1976), no. $1,55-135$.

[10] M. England and J. C. Eilbeck, Abelian functions associated with a cyclic tetragonal curve of genus six, J. Phys. A 42 (2009), 095210.

[11] J. C. Eilbeck, V. Z. Enolskii, and D. V. Leykin, On the Kleinian construction of Abelian functions of canonical algebraic curves, Proceedings of the Conference SIDE III: Symmetries of Integrable Differences Equations, Saubadia, May 1998, CRM Proceedings and Lecture Notes 25, 2000, pp. 121-138.

[12] J. C. Eilbeck, V. Z. Enolski, S. Matsutani, Y. Ônishi, and E. Previato, Abelian functions for trigonal curves of genus three, Int. Math. Res. Notices 2007 (2007), rnm 140-38.

[13] V. Enolski and J. Harnad, Schur function expansions of KP tau functions associated to algebraic curves, Russian. Math. Surveys, 2011, to appear, preprint 2008.

[14] J. D. Fay, Theta functions on Riemann surfaces, Lectures Notes in Mathematics (Berlin), vol. 352, Springer, 1973.

[15] J. D. Fay, Bilinear identities for theta functions, Mathematics Report 83-168, 1983.

[16] John Fay, Schottky Relations on $\frac{1}{2}(C-C)$, Proceedings of Symposia in Pure Mathematics, vol. 49:1, 1989, Theta Functions, Bowdoin 1987, pp. 485-501.

[17] F. Klein, Über hyperelliptische Sigmafunctionen, Math. Ann. 27 (1886), 431-464.

[18] F. Klein, Über hyperelliptische Sigmafunctionen, Math. Ann. 32 (1888), 351-380.

[19] B. G. Konopelchenko and G. Ortenzi, Algebraic curves in Birkhoff strata of Sato Grasmannian, arXiv: 1005.2053v1 [math-ph] 12 May 2010.

[20] I. M. Krichever, The method of algebraic geometry in the theory of nonlinear equations, Russian. Math. Surveys 32 (1977), 180-208.

[21] S. Matsutani and E. Previato, Jacobi inversion on strata of the Jacobian of the $C_{r s}$ curve, J. Math. Soc. Japan 60, 4, (2008), 1009-1044.

[22] S. Matsutani and E. Previato, Jacobi inversion on strata of the Jacobian of the $C_{r s}$ curve II, arXiv: 1006.1090 [math.AG]

[23] A. Nakayashiki, Algebraic Expression of Sigma Functions of $(n, s)$ Curves, arXiv:0803.2083, 2008.

[24] A. Nakayashiki, Tau and Sigma, Private communication, Iwate, 2008. 
[25] A. Nakayashiki, Sigma Function as A Tau Function Int. Math. Res. Notices, 2009, doi:10.1093/imrn/rnp135: arXiv:0904.0846 [math.AG].

[26] J. Nimmo, Symmetric functions and KP hierarchy In: Nonlinear evolution Equations, ed, J.J.P.Leon, (1982) pp. 245-261, World Scientific.

[27] B. E. Sagan, The Symmetric Group: Representations, Combinatorial Algorithms, and Symmetric Functions, Graduate Texts in Mathematics, 203, Springer, 2001.

[28] M. Sato and Y. Sato (Mori), On Hirota's bilinear equations I, RIMS Kokyuroku 338 (1980), 183.

[29] M. Sato and Y. Sato (Mori), On Hirota's bilinear equations II, RIMS Kokyuroku 414 (1981), 181.

[30] K. Weierstrass, Formeln und Lehrsätze zum Gebrauche der elliptischen Functionen, Springer, 1893, bearbeitet und herausgegeben von H. A. Schwarz.

Department of Mathematics and the Maxwell Institute for Mathematical Sciences, Heriot-Watt University, Edinburgh, UK EH14 4AS

E-mail address: J.C.Eilbeck@hw.ac.uk

Hanse-Wissenschaftskolleg, 27753 Delmenhorst, Germany,, On leave from Institute of Magnetism, National Academy of Sciences of Ukraine, Kyiv-142, UKRAINE

E-mail address: vze@ma.hw.ac.uk

Imperial College, 180 Queen's Gate, London SW7 2BZ

E-mail address: j.gibbons@ic.ac.uk 